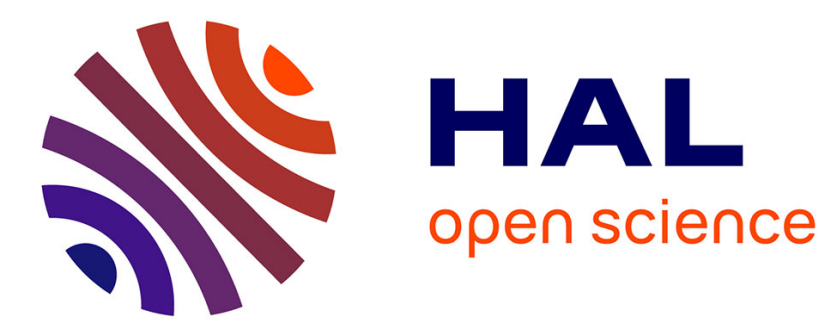

\title{
Use of global sensitivity analysis to assess the soil poroelastic parameter influence
}

Arnaud Mesgouez, Samuel Buis, Gaëlle Mesgouez, Gilles Micolau

\section{To cite this version:}

Arnaud Mesgouez, Samuel Buis, Gaëlle Mesgouez, Gilles Micolau. Use of global sensitivity analysis to assess the soil poroelastic parameter influence. Wave Motion, 2017, 72, pp.377-394. 10.1016/j.wavemoti.2017.04.001 . hal-01512234

\section{HAL Id: hal-01512234 \\ https://hal.science/hal-01512234}

Submitted on 12 Oct 2017

HAL is a multi-disciplinary open access archive for the deposit and dissemination of scientific research documents, whether they are published or not. The documents may come from teaching and research institutions in France or abroad, or from public or private research centers.
L'archive ouverte pluridisciplinaire HAL, est destinée au dépôt et à la diffusion de documents scientifiques de niveau recherche, publiés ou non, émanant des établissements d'enseignement et de recherche français ou étrangers, des laboratoires publics ou privés.

\section{(1) (1) $\$$}

Distributed under a Creative Commons Attribution - NonCommercial - NoDerivatives 44.0 


\title{
Use of global sensitivity analysis to assess the soil poroelastic parameter influence
}

\author{
A. Mesgouez ${ }^{\mathrm{a}, \mathrm{b}, *}$, S. Buis ${ }^{\mathrm{b}, \mathrm{a}}$, G. Lefeuve-Mesgouez ${ }^{\mathrm{a}, \mathrm{b}}, \mathrm{G}$. Micolau ${ }^{\mathrm{a}, \mathrm{b}}$ \\ a UMR 1114 EMMAH, Université d'Avignon et des Pays de Vaucluse, Campus Jean-Henri Fabre, Agroparc, 201 rue Baruch de Spinoza, \\ BP 21239, F-84916 Avignon Cedex 9, France \\ b UMR 1114 EMMAH, INRA, Domaine Saint Paul - Site Agroparc, F-84914 Avignon Cedex 9, France
}

- A global sensitivity analysis applied to poroelastic wave propagation is presented.

- The most influential porous parameters for a soil configuration are investigated.

- Partial variances are determined with the Extended FAST method.

- A specific focus on the interactions between parameters is proposed.

In this study, we show how a global sensitivity analysis method can be used to obtain relevant information for the interpretation of the mechanical wave propagation phenomena involved in a poroelastic soil that takes into account the presence of water. The present investigation addresses the issue of the identification and the ranking of the most influential parameters. The sensitivity indices provide a variance-based measure of the uncertainty effects of the input parameters on the mechanical outputs of the model. It allows quantification of, on the one hand, the influence of each parameter and on the other hand, the possible interactions between all the parameters. Numerical simulations are performed in a laboratory-scale configuration: a fluid medium overlying a poroelastic material is submitted to a transient excitation, and the coupling of the acoustic and Biot models is solved using a semi-analytical approach. The analysis of the temporal and spatial evolution of partial variances highlights the most important parameters and the complementary information contained in the signals in function of both the time and the receiver location. In particular, we show that the description of the poroelastic waves is governed by only a restricted number of parameters for the configuration under study.
Keywords:

Global sensitivity analysis

Poroelastic parameters

Biot model

Sobol indices

Wave propagation

EFAST method

\section{Introduction}

The present investigation addresses the issue of the use of a global sensitivity analysis method applied to a poroelastic wave propagation problem. Obtaining structural images of a porous material, and estimating its physical properties, is a

\footnotetext{
* Corresponding author at: UMR 1114 EMMAH, Université d'Avignon et des Pays de Vaucluse, Campus Jean-Henri Fabre, Agroparc, 201 rue Baruch de Spinoza, BP 21239, F-84916 Avignon Cedex 9, France.

E-mail addresses: arnaud.mesgouez@univ-avignon.fr (A. Mesgouez),samuel.buis@paca.inra.fr (S. Buis),gaelle.mesgouez@univ-avignon.fr

(G. Lefeuve-Mesgouez), gilles.micolau@univ-avignon.fr (G. Micolau).
} 
topic of considerable interest in many branches of activities in geophysics, underwater acoustics, civil engineering, and biomechanics [1-8].

One of the most important problems in the characterization of porous materials is the large number of unknown parameters and their associated uncertainties, which makes the inversion process tedious and difficult [1,3,9-13]. In this context, sensitivity analysis (SA), i.e. the study of the impact of model inputs on model outputs, may provide relevant information about the relationships between uncertain model input parameters and model outputs. This physical insight into models of wave propagation in heterogeneous media may help in a second stage in setting up a subtler strategy: (i) to characterize the material by identifying the main parameters controlling the variability of the model output, or to modify the model itself by a reduction of the influential input parameters; (ii) to help construct experimental designs for model inversion.

SA has always been practiced since the existence of simulation models, often using simple empirical techniques such as perturbing a given input and analyzing the impact of this perturbation on model outputs using simple graphical or numerical representations of the gap between perturbed and baseline simulations. However, it has become increasingly popular over the last twenty years with the development of both new techniques, computational power and solid mathematical background. Many different methods are now available for SA [14] and the development of new methods, improving computational efficiency, or description of model behavior, or relaxing the need of restrictive hypotheses is still a dynamic research field [15]. SA methods are traditionally divided into two families: local and global methods [16]. Local methods study the local impact of model inputs on model outputs. In this framework, inputs often vary one at a time (when one input is varied, the others are held constant) within a small interval around a nominal value. These methods are computationally easy to implement but the volume of the explored input space is very limited, which prevents from a full description of the model behavior. Global sensitivity analysis (GSA) methods incorporate the influence of the whole range of variation of model inputs and often evaluate the impact of the inputs while all the other parameters can vary. Among global methods, variancebased methods are very popular. Their principle is to apportion the total variance of model outputs to the various input factors and to their interactions, given their uncertainty distributions. They are model-independent and they can deal with nonlinear models. To the best of our knowledge, GSA techniques have, however, still rarely been applied to wave propagation in poroelastic environments.

In this study, both temporal evolution for specific simulated sensors, and spatial description for a given time, of the sensitivity indices are analyzed to highlight the most influential mechanical parameters, and the field and period of influence. The poroelastic 2D laboratory-scale configuration under study is a combination of two half-spaces. The upper half-space is a purely acoustic medium, in which a source point emits temporal cylindrical waves. The excitation term, the quality of the interface, and the acoustical parameters are supposed to be perfectly well known a priori. The lower half-space is a poroelastic material, described by the low-frequency Biot partial differential equation solved with a semi-analytical approach [17,18]. The mechanical parameters in this region are uncertain and assumed independent. It is supposed in this paper that their uncertainties are described by a uniform law, considering that the range of uncertainties for each parameter covers $\pm 10 \%$ of their presumed value $[12,19]$. Mechanical output fields of interest are the solid velocity vertical component and the pore pressure. The full waveform response is considered. The poroelastic configuration under study respects the three following requirements: (i) it is of interest to various applications cited before; (ii) the semi-analytical approach, which is the kernel of the forward problem used for performing the global sensitivity analysis, has already been crosschecked and published $[17,18]$, and (iii) the relative code provides a running fast model, that can be used for a large number of model evaluations required by the GSA within a reasonable computational time.

The remainder of this paper is organized as follows. In Section 2, the variance-based sensitivity indices are described. The concepts and practical implementation of the EFAST method used to estimate these indices are described in Section 3. In Section 4, the geometry, methodology, and numerical values are stated. Results of the GSA both in time and space are discussed in Section 5, in which the most relevant and influential parameters are identified. The latter section ends with comments on the genericity aspects of the study. Concluding remarks and perspectives are presented in Section 6.

\section{Variance-based sensitivity indices}

Sensitivity indices are variance-based: they express the partial variances of the model output that can be apportioned to the various model inputs. We specify in the following how the functional decomposition of a model can be used, by considering model inputs in a probabilistic space and under given hypotheses, to decompose the variance of the model output into partial variances.

\subsection{Functional ANOVA decomposition}

Let us consider a model represented as a function $y=f(\mathbf{x})$, with $\mathbf{x} \in D \subset \mathbb{R}^{k}$ the vector of its $k$ scalar input variables and $y \in \mathbb{R}$ its unique scalar output variable (the case of multiple model outputs is discussed at the end of Section 2.3).

Each function $f$ defined and integrable on $D$ can be decomposed in a unique way [20]:

$$
f(\mathbf{x})=f_{0}+\sum_{i} f_{i}\left(x_{i}\right)+\sum_{1 \leqslant i<j \leqslant k} f_{i j}\left(x_{i}, x_{j}\right)+\cdots+f_{12 \ldots k}\left(x_{1}, \ldots, x_{k}\right)
$$

where functions $f_{*}$ are mutually orthogonal on $D$ for the inner-product $\langle f \mid g\rangle=\int_{D} f(\mathbf{x}) \cdot g(\mathbf{x}) d \mathbf{x}$. 
This decomposition is often called ANOVA-decomposition or High-Dimensional-Model-Representations (HDMR) [21]. It splits the function $f$ into terms representing individual contributions of the different input variables and terms, often called interaction terms, dependent on multiple input variables.

\subsection{Variance decomposition}

Let us now suppose that the vector of input variables $\mathbf{x}$ is unknown and may be represented by a vector of random variables $\mathbf{X}$ following a given joint probability distribution $\pi$ modeling its uncertainty. The random variables $X_{i}$ associated to the model input variables $x_{i}$ are often called factors in sensitivity analysis. It follows that $Y=f(\mathbf{X})$ is also a random variable. The functional decomposition presented in Eq. (1) can be generalized to any probability law $\pi$ on $D$ by considering the inner-product $\langle f \mid g\rangle=\int_{D} f(\mathbf{x}) \cdot g(\mathbf{x}) \cdot \pi(\mathbf{x}) d \mathbf{x}$. The different terms of the functional decomposition can then be expressed in the function of conditional expectations using their orthogonal properties:

$$
\begin{aligned}
& f_{0}=\mathbb{E}(Y) \\
& f_{i}\left(x_{i}\right)=\mathbb{E}\left(Y \mid X_{i}=x_{i}\right)-f_{0} \\
& f_{i j}\left(x_{i}, x_{j}\right)=\mathbb{E}\left(Y \mid X_{i}=x_{i}, X_{j}=x_{j}\right)-f_{0}-f_{i}\left(x_{i}\right)-f_{j}\left(x_{j}\right) \\
& \ldots
\end{aligned}
$$

where $\mathbb{E}\left(Y \mid X_{i}=x_{i}\right)$ is the conditional expectation of $Y$ given $X_{i}=x_{i}$ and $\mathbb{E}\left(Y \mid X_{i}=x_{i}, X_{j}=x_{j}\right)$ is the conditional expectation of $Y$ given $X_{i}=x_{i}$ and $X_{j}=x_{j}$. In the following, notations $\mathbb{E}\left(Y \mid X_{i}\right)$ and $\mathbb{E}\left(Y \mid X_{i}, X_{j}\right)$ are used instead of $\mathbb{E}\left(Y \mid X_{i}=x_{i}\right)$ and $\mathbb{E}\left(Y \mid X_{i}=x_{i}, X_{j}=x_{j}\right)$ for the sake of brevity.

At this stage, two important hypotheses have to be considered:

- probability distributions of factors $X_{i}$ are independent,

- function $f$ is square-integrable on $D$, and as a consequence, all functions $f_{*}$ are square-integrable on $D$.

The hypothesis concerning the independence of the $X_{i}$ distributions can be restrictive in practice. This point is discussed at the end of Section 5.

Considering (1) and (2), the variance of $Y=f(\mathbf{X})$ can then be expressed as:

$$
\begin{aligned}
& \mathbb{V}(Y)=\sum_{i} V_{i}+\sum_{1 \leqslant i<j \leqslant k} V_{i j}+\cdots+V_{1,2, \ldots, k} \\
& \text { with } \\
& V_{i}=\mathbb{V}\left(\mathbb{E}\left(Y \mid X_{i}\right)\right) \\
& V_{i j}=\mathbb{V}\left(\mathbb{E}\left(Y \mid X_{i}, X_{j}\right)\right)-V_{i}-V_{j} \\
& \cdots
\end{aligned}
$$

The variance of $Y$ can thus be expressed as a sum of individual contributions of the different factors and of their interactions.

\subsection{Main and total effects}

$V_{i}$ is the partial variance of $Y$ attributed solely to $X_{i}$, i.e. explained by the variations of $X_{i}$ alone independently of the variations of the other factors. $V_{i}$ is often called main or principal effect of $X_{i}$.

$V_{i j}$ is the partial variance of $Y$ attributed to the second order effect of $X_{i}$ and $X_{j}$, i.e. it can be explained by the variations of $X_{i}$ and $X_{j}$ on their uncertainty domains but not by the sum of their main effects. It describes the interaction between $X_{i}$ and $X_{j}$, i.e. the fact that the effect of $X_{i}\left(\right.$ resp. $\left.X_{j}\right)$ may depend on the values of $X_{j}\left(\right.$ resp. $\left.X_{i}\right)$.

As computing $\left(2^{k}-1\right)$ partial variances of decomposition (3) is practically often intractable, [22] introduced the concept of total-effect. The partial variance $V T_{i}$ attributed to the total effect of $X_{i}$ is:

$$
V T_{i}=V_{i}+\sum_{1 \leqslant j \neq i \leqslant k} V_{i j}+\cdots+V_{1,2, \ldots, k}
$$

It includes the effect of $X_{i}$ alone as well as interactions with any combination of other factors. The difference between $V T_{i}$ and $V_{i}$ is the partial variance attributed to the interactions of all orders between $X_{i}$ and the other factors. Moreover, from (3) and (4), we have:

$$
V T_{i}=\mathbb{V}(Y)-\mathbb{V}\left(\mathbb{E}\left(Y \mid X_{\sim i}\right)\right)
$$

where $\sim i$ stands for "all indices except $i$ ". $\mathbb{V}\left(\mathbb{E}\left(Y \mid X_{\sim i}\right)\right)$ is the sum of the partial variances attributed to the effects of all orders implying all factors except $X_{i}$.

All these partial variances are defined in $[0, \mathbb{V}(Y)]$. If $\sum_{i} V_{i}=\mathbb{V}(Y)$ then the model is said to be additive whereas $\mathbb{V}(Y)-\sum_{i} V_{i}>0$ indicates the presence of interaction effects. Estimation of the $k$ pairs $\left(V_{i}, V T_{i}\right)$ is often performed in practice since it yields a good and synthetic, although non exhaustive, characterization of the sensitivity pattern for a model, as mentioned by [16]. These sensitivity indices allow to address several GSA settings as discussed in [23]. 
In the literature, variance-based sensitivity analyses are usually presented using Sobol indices. These indices, introduced in [20], have been widely used these last twenty years for sensitivity analysis studies. They correspond to the normalization of the partial variances $V_{i}, V_{i j}, \ldots, V_{1,2, \ldots, k}$ and $V T_{i}$ through the total variance $\mathbb{V}(Y)$. In our study, we have chosen to use partial variances instead of Sobol indices because we have to handle multivariate model outputs. Handling sensitivity of multivariate model outputs such as dynamic or spatial variables can be achieved in several ways [24]. Graphical representation of dynamic (resp. spatial) evolution of sensitivity indices allows having an exhaustive view of the factor importance on the selected variable and of their temporal or spatial variability. In this context, using partial variances is useful to directly appreciate the variations in time and space of the response uncertainty whereas Sobol indices naturally hide this information owing to the normalization. The use of Sobol indices may thus highlight important parameters at some period and/or spatial area where this uncertainty is very small.

\section{Extended FAST}

The computation of partial variances $V_{i}$ does not raise any particular issue and many methods are available to compute them. Estimating the total effects of $V T_{i}$ requires ad hoc numerical experimental design and associated estimators. Two families of methods are actually available for computing both $V_{i}$ and $V T_{i}$ : the so-called Sobol and FAST methods. They typically require several dozen thousands of model simulations for obtaining precise estimates for partial variances. The Fourier Amplitude Sensitivity Test (FAST) method has been introduced by [25-27]. Then, [28-30] proposed some implementations and further improvements but these first versions were, however, only able to estimate first order indices. Saltelli et al. [31] proposed a set of extensions to the classical FAST method introducing the possibility of computing total indices leading to the so-called Extended-FAST (EFAST) method. Continuous improvements were proposed the last 15 years: extension to non-uniformly distributed factors [32,33]; computation of first-order indices for correlated factors [34,35]; improvements of computing efficiency for first [35-37] and higher order indices [38,39]. The FAST method is considered to be robust [38], and is very popular since Saltelli and colleagues recommended its use [40,41]. The reader can refer for examples to [33] or [35] for short reviews of studies using this method. Different comparisons with the Sobol method have shown that the FAST approach is less computationally demanding for equivalent precision [31,41,42].

\subsection{Concepts and practical implementation}

The main principle of the FAST method is to make the model input variables oscillating at different frequencies and to assess the importance of the variables by scrutinizing the Fourier coefficients of the model output at some specific frequencies. For that, a variable substitution $f(\mathbf{x})=f\left(G_{1}(s), G_{2}(s), \ldots, G_{k}(s)\right)$ is performed with:

$$
x_{i}=G_{i}\left(\sin \left(\omega_{i} s+\varphi_{i}\right)\right) \quad \forall i=1,2, \ldots, k
$$

where $s$ is a scalar variable, $\omega_{i}$ is the integer frequency associated to variable $x_{i}, G_{i}$ is the transformation function, and $\varphi_{i}$ is a random phase-shift chosen uniformly in $[0,2 \pi]$, which allows a change in the starting point of the scheme.

The variations of $s$ within $[-\pi, \pi]$ make all variables vary simultaneously in order to explore the input space. The multidimensional integrals on vector $\mathbf{x}$ necessary for the computation of variances and conditional expectations (Eqs. (3) and (5)) are transformed into more easily tractable one-dimension integrals on scalar $s$ [25]. An appropriate choice for functions $G_{i}$ is however necessary so that variations in $s$ make variables $x_{i}$ varying following the probability distributions of factors $X_{i}$. Lu and Mohanty [32] proposed to use $G_{i}(z)=F_{i}^{-1}(1 / \pi \arcsin (z)+1 / 2)$, with $F_{i}^{-1}$ the inverse cumulative distribution function of the probability distribution of $X_{i}$. The values of the variables $x_{i}$ are thus computed using:

$$
x_{i}=F_{i}^{-1}\left(\frac{1}{\pi} \arcsin \left(\sin \left(\omega_{i} s+\varphi_{i}\right)\right)+\frac{1}{2}\right), \quad \forall i=1,2, \ldots, k
$$

with $\varphi_{i}$ randomly chosen in $[0,2 \pi]$ (see Eq. $(6)$ ), and $F^{-1}(p)=a+p(b-a)$ for uniform distributions of support $[a, b]$ and $0<p<1$.

Using integer frequencies $\omega_{i}$ implies that variables $x_{i}$ oscillate periodically at frequency $\omega_{i}$ and function $f$ to be a periodical function of $s$ with a period of at most $2 \pi . f$ can be thus expanded into Fourier series over the domain of integer frequencies $l \in \mathbb{Z}$ :

$$
f(\mathbf{x})=\sum_{l=-\infty}^{+\infty}\left(A_{l} \cos (l s)+B_{l} \sin (l s)\right)
$$

where $A_{l}$ and $B_{l}$ are Fourier coefficients defined as:

$$
\begin{aligned}
& A_{l}=\frac{1}{2 \pi} \int_{-\pi}^{\pi} f(s) \cos (l s) \mathrm{d} s \\
& B_{l}=\frac{1}{2 \pi} \int_{-\pi}^{\pi} f(s) \sin (l s) \mathrm{d} s
\end{aligned}
$$


The spectrum of the Fourier series expansion is defined as $\wedge_{l}=A_{l}^{2}+B_{l}^{2}$ and it characterizes the amplitude of $f$ at frequency l. It can be shown that total variance $\mathbb{V}(Y)$ (Eq. (3)) can be expressed as the sum of the elements of the Fourier spectrum of $f[25]$ :

$$
\mathbb{V}(Y)=2 \sum_{l=1}^{+\infty} \wedge_{l}
$$

If factor $X_{i}$ has a strong impact on the model output, the oscillations of $f(\mathbf{x})$ at frequency $\omega_{i}$ and associated harmonics (i.e. frequencies $p \omega_{i}$, with $p$ being a non-null integer) should be of high amplitude. The importance of the Fourier component of $f$ associated to $X_{i}$ is quantified by summing the elements of the spectrum corresponding to this frequency and its associated harmonics. The partial variance of $Y$ attributed to the main effect of $X_{i}$ is thus obtained by:

$$
V_{i}=2 \sum_{p=1}^{+\infty} \wedge_{p \omega_{i}}
$$

In practice the infinite summation is not convenient. Because $f$ is square-integrable, it is expected that the amplitude of the spectrum at the harmonic $p \omega_{i}$ decays with $p$ so that, after a certain number of harmonics (say $M$ ), the contribution of high-order terms to the total variance are negligible (albeit with some interference/aliasing effects). The main effect of $X_{i}$ can thus be approximated by:

$$
\hat{V}_{i}=2 \sum_{p=1}^{M} \Lambda_{p \omega_{i}}
$$

$M$ is called the interference factor and is usually chosen between [4,6].

The authors in [31] further proposed a heuristic, recently corroborated by [43], to compute the total effect of $X_{i}$ by setting a high value to $\omega_{i}$ (typically $\omega_{i}=2 M \max \left(\boldsymbol{\omega}_{\sim i}\right)$ ) and low ones to the other frequencies (gathered in the vector of frequencies $\left.\omega_{\sim i}\right) . \mathbb{V}\left(\mathbb{E}\left(Y \mid X_{\sim i}\right)\right)$, the sum of the partial variances attributed to the effects of all orders implying all factors except $X_{i}$ (Eq. (5)), is then computed by summing the spectrum elements of all frequencies between 1 and $M \max \left(\omega_{\sim i}\right)$, assuming that interaction effects implying $\omega_{i}$ are negligible at these frequencies. The total variance and the partial variance of $Y$ attributed to the total effect of $X_{i}$ are thus obtained by:

$$
\hat{V}=2 \sum_{l=1}^{M \omega_{i}} \wedge_{l}
$$

and

$$
\hat{V}_{i}=2 \sum_{l=1}^{M \omega_{i}} \wedge_{l}-2 \sum_{l=1}^{M \max \left(\boldsymbol{\omega}_{\sim i}\right)} \wedge_{l}=2 \sum_{l=M \max \left(\boldsymbol{\omega}_{\sim i}\right)+1}^{M \omega_{i}} \wedge_{l}
$$

As a consequence, the minimum number of model runs to limit the problem of aliasing is $N=2 M \omega_{i}+1$ according to the Nyquist-Shannon theorem. Better results might however be obtained using $N=4 M \omega_{i}+1$ [31]. The key elements for an efficient estimate of the partial variances are the choice of the interference factor $M$ and frequency set $\omega_{\sim i}$.

The authors in [31] proposed an algorithm to compute these frequency sets $\omega_{i}$ and $\boldsymbol{\omega}_{\sim i}$ and the partial variances $\hat{V}_{i}$ and $\hat{V} T_{i}$ provided the number of factors $k$, the sample size $N$ and the interference factor $M$. This algorithm is described in detail in Appendix A. In this study we used a Matlab implementation of this algorithm [44]. An R implementation can be found in the widely used package "sensitivity" [45] available on the CRAN.

A global scheme describing the different steps necessary for the computation of the partial variances is presented in Fig. 1. It must be noted that they can be computed independently for each model output variables (or for each time step or spatial point of a given model output variable) at no extra-cost by using the same model simulations.

\subsection{Configuration of use in this study}

The choice of $N, M$, and of the Nyquist constraint has been determined in a preliminary study conducted on a restricted model configuration ( 1 spatial point) limiting the amount of stored data and of associated post-treatments. Several values have been experimented for $M(4$ and 6$), N\left(257,513,1025\right.$, and 2049), and for the Nyquist constraint $\left(N=2 M \omega_{i}+1\right.$ and $\left.N=4 M \omega_{i}+1\right)$. Each sensitivity analysis has been repeated 5 times by changing the starting point of the search-curve using random values of $\varphi_{i}$. A dummy parameter, that does not appear in the model equations, has been considered to test the significance of the estimated parts of variance [46]. Final estimates of $\widehat{V}_{i}$ and $\widehat{V T}_{i}$ have been computed by taking the averages of the values obtained for the different repetitions. 211420 simulations were performed for conducting this preliminary study. The final choice $\left(N=1025, M=4\right.$ and $\left.N=2 M \omega_{i}+1\right)$ has been determined by (i) considering the convergence of the $\widehat{V}_{i}$ and $\widehat{V T}_{i}$ values and of the parameter rankings, (ii) checking that the sum of the $\widehat{V}_{i}$ is not larger than $\widehat{V}$, and (iii) checking that the $\widehat{V}_{i}$ and $\widehat{V T}_{i}$ values of the dummy parameter are close to zero. A new study using these selected values has been then conducted on the model in its full spatial configuration (see next Section). The total cost of this new analysis was $5 N k=51250$ model simulations. 


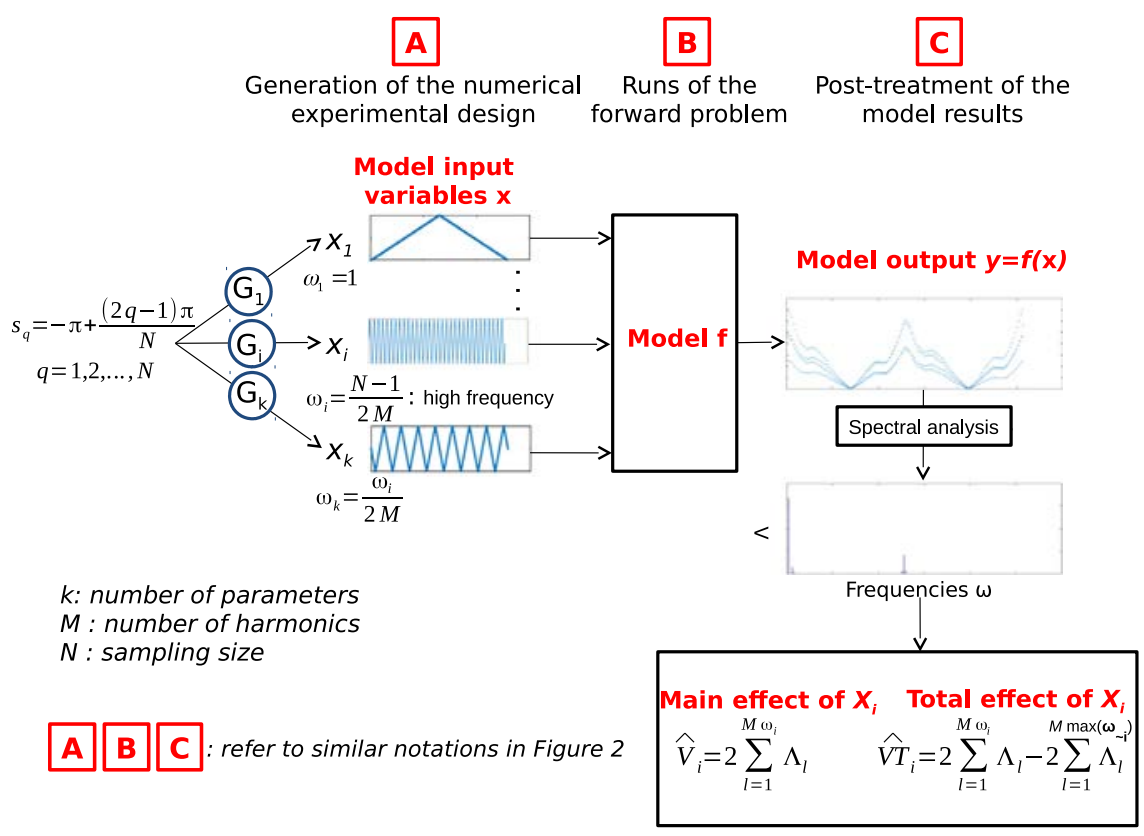

Fig. 1. Synthetic presentation for the estimation of partial variances $\hat{V}_{i}$ and total effects $\hat{V}_{i}$ of factor $X_{i}(i=1,2, \ldots, k)$.

\section{Model and configuration under study}

Fig. 2 presents a graphical description summarizing the methodology. The use of the EFAST method generates a numerical experimental design. With these sets of uncertain parameter values, the direct problem is performed, and results are postprocessed to obtain the variances and sensitivity indices. This approach emphasizes the main influence parameters.

The configuration presented below has been chosen to illustrate the methodology because:

- it is of interest to applications in the field of underwater acoustics, material wave characterization, or civil engineering for natural or artificial media [1-8],

- the semi-analytical approach, which is the kernel of the forward problem used for performing the global sensitivity analysis, has already been crosschecked and published [17,18],

- the computational cost of the semi-analytical approach allows for numerous repetitions.

\subsection{Geometry}

The 2D configuration under investigation is a fluid half-space $\Omega_{F}$ above a homogeneous poroelastic half-space $\Omega_{P}$, as shown in Fig. 3. The $x$ and $y$ geometrical axes point rightward and upward, respectively. A causal source point $S(t)$ located in the fluid at $\left(x_{s}=0, y_{s}>0\right)$ emits cylindrical waves. The expression of $S(t)$ is given in Appendix B.1.

\subsection{Physical model: inputs and outputs}

The fluid domain $\Omega_{F}$ is governed by the acoustic equations, recalled in Appendix B.2. The physical parameters are the density $\rho_{F}$ and the bulk modulus $K_{F}$ supposed to be perfectly known.

The poroelastic medium $\Omega_{P}$ is modeled using the low-frequency Biot theory [47-49], recalled in Appendix B.3. It corresponds to the medium to be investigated. The 10 uncertain physical parameters of the Biot theory are:

- the dynamic viscosity $\eta$, the bulk modulus $K_{F}$, and the density $\rho_{F}$ of the saturating fluid; notations $\rho_{F}$ and $K_{F}$ are used both in $\Omega_{P}$ and $\Omega_{F}$ but only those concerning $\Omega_{P}$ are supposed to be uncertain, which, from a physical point of view, takes into account a partial saturation of the porous material,

- the density $\rho_{S}$, the bulk modulus $K_{S}$, and the shear modulus $\mu$ of the elastic skeleton,

- the connected porosity $\phi$, the tortuosity $a_{\infty}$, the absolute permeability $\kappa$, and the bulk modulus of the dry isotropic matrix $K_{M}$. 


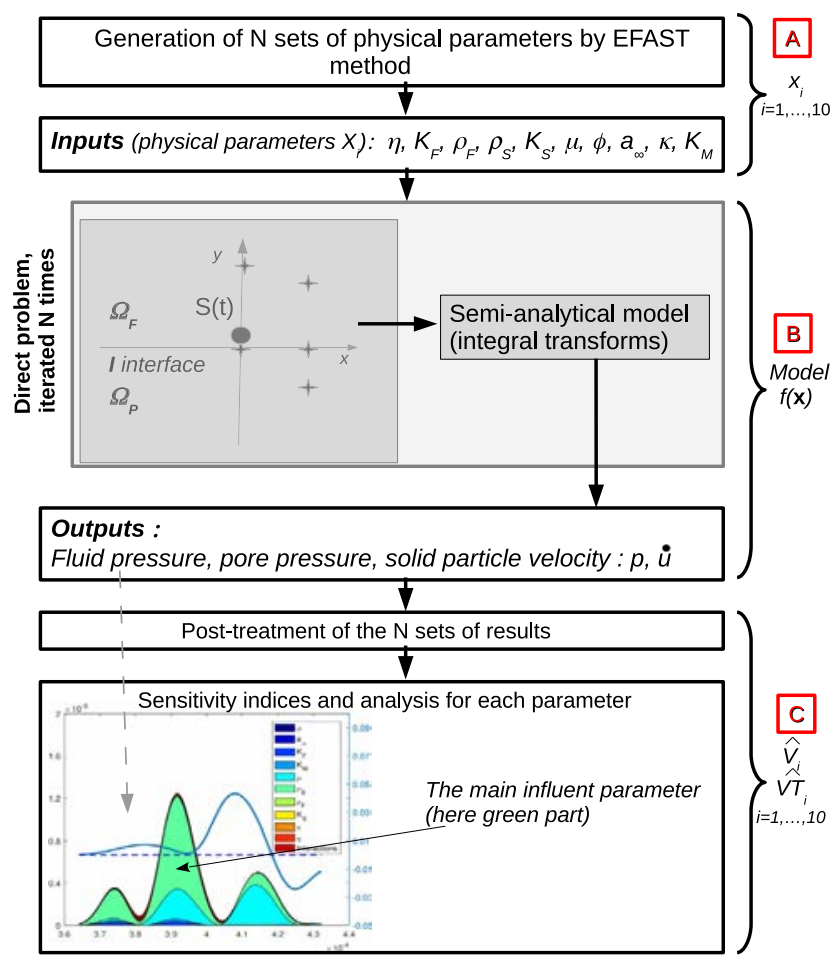

Fig. 2. Methodology applied for the global sensitivity analysis (GSA).

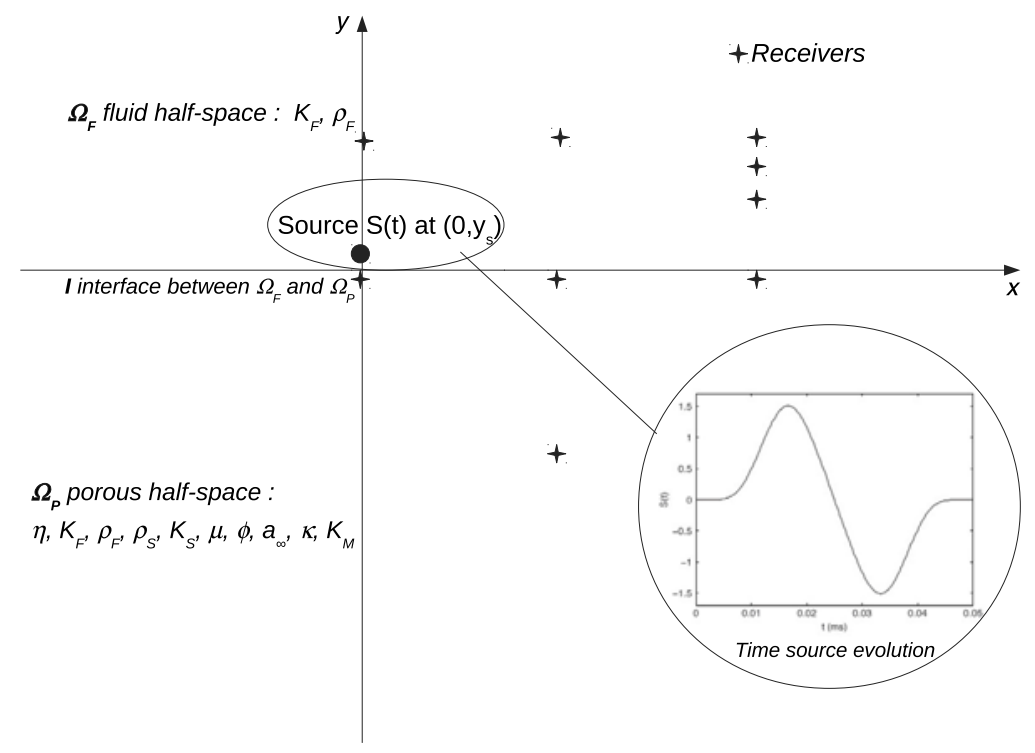

Fig. 3. Configuration under study : fluid medium $\Omega_{F}$ over a homogeneous poroelastic half-space $\Omega_{P}$.

The fluid/porous interface $I$ has been chosen in this study to be modeled as an "open-pore" interface [48,50,51], recalled in Appendix B.4. Other interface conditions could be taken into account [17] but this is not under the scope of application of the present work.

The inputs of the global sensitivity analysis study are the 10 porous parameters. The range of variations is given in Section 4.3. The outputs of interest are: the acoustic pressure $p$ in $\Omega_{F}$, the solid velocity $\dot{\mathbf{u}}=\left(\dot{u}_{x}, \dot{u}_{y}\right)^{T}$ and the pore pressure $p$ in $\Omega_{P}$. The overlying dot denotes the partial derivative with respect to time and $\mathbf{u}$ is the solid displacement. 
Table 1

Mean (or targeted) physical parameters and acoustic properties relative to the mean poroelastic half-space.

\begin{tabular}{lll}
\hline Saturating fluid & $\rho_{F}\left(\mathrm{~kg} / \mathrm{m}^{3}\right)$ & 1000 \\
& $K_{F}(\mathrm{~Pa})$ & $2.2510^{9}$ \\
& $\eta(\mathrm{Pa} \mathrm{s})$ & $10^{-3}$ \\
\multirow{3}{*}{ Grain } & $\rho_{S}\left(\mathrm{~kg} / \mathrm{m}^{3}\right)$ & 2760 \\
& $\mu(\mathrm{Pa})$ & $3.4010^{9}$ \\
& $K_{S}(\mathrm{~Pa})$ & $4.010^{10}$ \\
Matrix & $\phi$ & 0.24 \\
& $a_{\infty}$ & 2.3 \\
& $\kappa\left(\mathrm{m}^{2}\right)$ & $3.910^{-13}$ \\
& $K_{M}(\mathrm{~Pa})$ & $5.810^{9}$ \\
\hline
\end{tabular}

Table 2

Characteristic velocities (at $f_{C}=20 \mathrm{kHz}$, central frequency of the source term); $c_{P f}$ and $c_{P S}$ are the two compressional poroelastic wave celerities, $c_{S}$ is the shear poroelastic wave celerity and $c_{F}$ is the fluid wave celerity.

\begin{tabular}{|c|c|c|}
\hline \multirow[t]{4}{*}{ Phase velocities } & $c_{P f}\left(f_{C}\right)(\mathrm{m} / \mathrm{s})$ & 2636.8 \\
\hline & $c_{P_{S}}\left(f_{C}\right)(\mathrm{m} / \mathrm{s})$ & 571.5 \\
\hline & $c_{S}\left(f_{C}\right)(\mathrm{m} / \mathrm{s})$ & 1210.8 \\
\hline & $c_{F}(\mathrm{~m} / \mathrm{s})$ & 1500.0 \\
\hline
\end{tabular}

The approach uses a direct model based on integral transforms. Details are given in [17,18]. Appendix C only presents the main characteristics of the semi-analytical approach.

\subsection{Numerical values}

The excitation source $S(t)$ is located in the fluid domain near the interface at point $\left(x_{s}=0, y_{s}=4.010^{-3}\right) \mathrm{m}$. The source emits a combination of sinusoids of central frequency $20 \mathrm{kHz}$ during the time period $t \in\left[0,5.10^{-5}\right] \mathrm{s}$.

The acoustic medium $\Omega_{F}$ is water with $\rho_{F}=1000 \mathrm{~kg} / \mathrm{m}^{3}$ and $K_{F}=2.2510^{9} \mathrm{~Pa}$. The poroelastic half-space $\Omega_{P}$ consists of water-saturated sand [52]. Table 1 gives the 10 mean (or targeted) values of the poroelastic parameters. The EFAST analysis is conducted with random parameters uniformly distributed with a $\pm 10 \%$ variation around these mean values, used for instance in [12] or [19].

Values for the shear wave speed and the low and fast wave speeds are given in Table 2.

\section{Results and discussion}

Results concern first receivers located in the upper fluid part $\Omega_{F}$, then at the fluid/porous medium interface I and, at the end, in the porous medium $\Omega_{P}$. For points located in the fluid, we focus on the full waveform pressure $p$ response whereas for points located in the porous medium, we focus both on the full waveform pressure $p$ and the vertical component of the solid velocity $\dot{u}_{y}$.

\subsection{Upper fluid part $\Omega_{\mathrm{F}}$}

Fig. 4 shows the temporal evolution of the partial variances for fluid pressure at the receiver located at $(x=0.3 ; y=0.3)$ $\mathrm{m}$. The temporal fluctuation of the total variance due to the variation of the 10 studied parameters in their uncertainty domain corresponds to the sum of the colored contribution of each parameter and of their interaction. The temporal variation of the fluid pressure mean (blue curve) indicates that variances significantly different from zero are concentrated on the wave passage, i.e. centered around $t=d / c_{F}+t_{C} / 2 \approx 0.31 \mathrm{~ms}$ in this case. This behavior has been observed at every spatial point studied although the time domain of the wave passage obviously differs with the point location and medium under study. Thus, only a restricted temporal part of the signal corresponding to the time domain for which the total variance is significantly different from zero are shown in the Figures (we have selected, for all the results, the temporal part of the simulated measure for which the variance is higher than one percent of the variance maximum value).

On Fig. 4, the variance reaches a value of $5 \cdot 10^{-6} \mathrm{~Pa}$, which gives a standard deviation of about $2.10^{-3} \mathrm{~Pa}$ compared to the maximum value of the fluid pressure mean of $8.10^{-2} \mathrm{~Pa}$. This corresponds to a $2.5 \%$ fluctuation. A direct inspection of the results reveals that for this chosen point, the variance is mainly explained by four parameters $: \rho_{S}, \phi, K_{F}$, and $\mu$. The strong contrast between the fluid and solid densities, and the location of the source near the interface, highlight the impact of parameter $\rho_{s}$. Other parameters have really minor contributions.

Figs. 5 and 6 present, respectively, the partial variances for fluid pressure at various $x(0.1 ; 0.2 ; 0.4 ; 0.5) \mathrm{m}$ but at a fixed elevation $y=0.3 \mathrm{~m}$, and at various $y(0.1 ; 0.2 ; 0.4 ; 0.5) \mathrm{m}$ but at a fixed abscissa value $x=0.3 \mathrm{~m}$. The same scales are used for the variance and the pressure mean values on all the subfigures. 


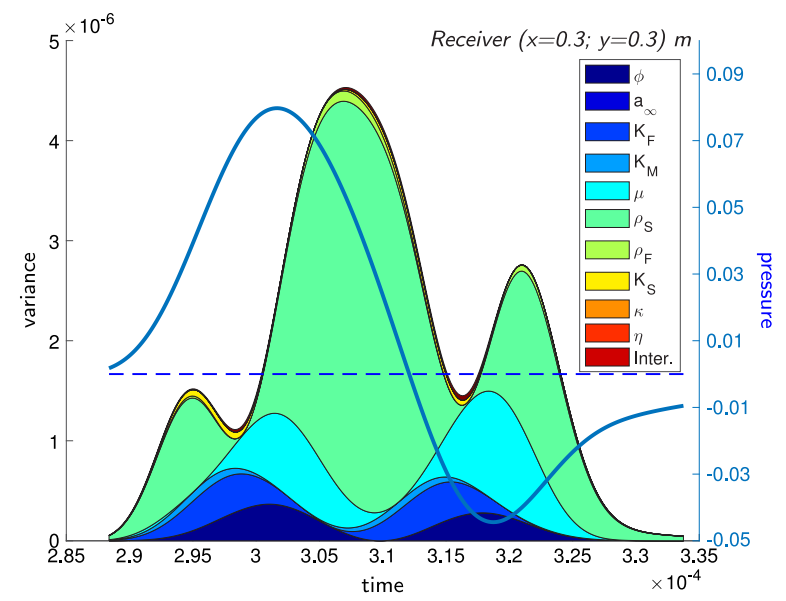

Fig. 4. Full time (s) evolution of the partial variances for fluid pressure ( $\mathrm{Pa})$ at a selected location $(x=0.3, y=0.3) \mathrm{m}$ in $\Omega_{\mathrm{F}}$. Total variance is the sum of the colored contribution of each parameter and of their interaction. The blue curve represents the full time response of the fluid pressure mean. Only the temporal part of the simulated measure for which the variance is higher than one percent of the variance maximum value has been drawn. (For interpretation of the references to color in this figure legend, the reader is referred to the web version of this article.)
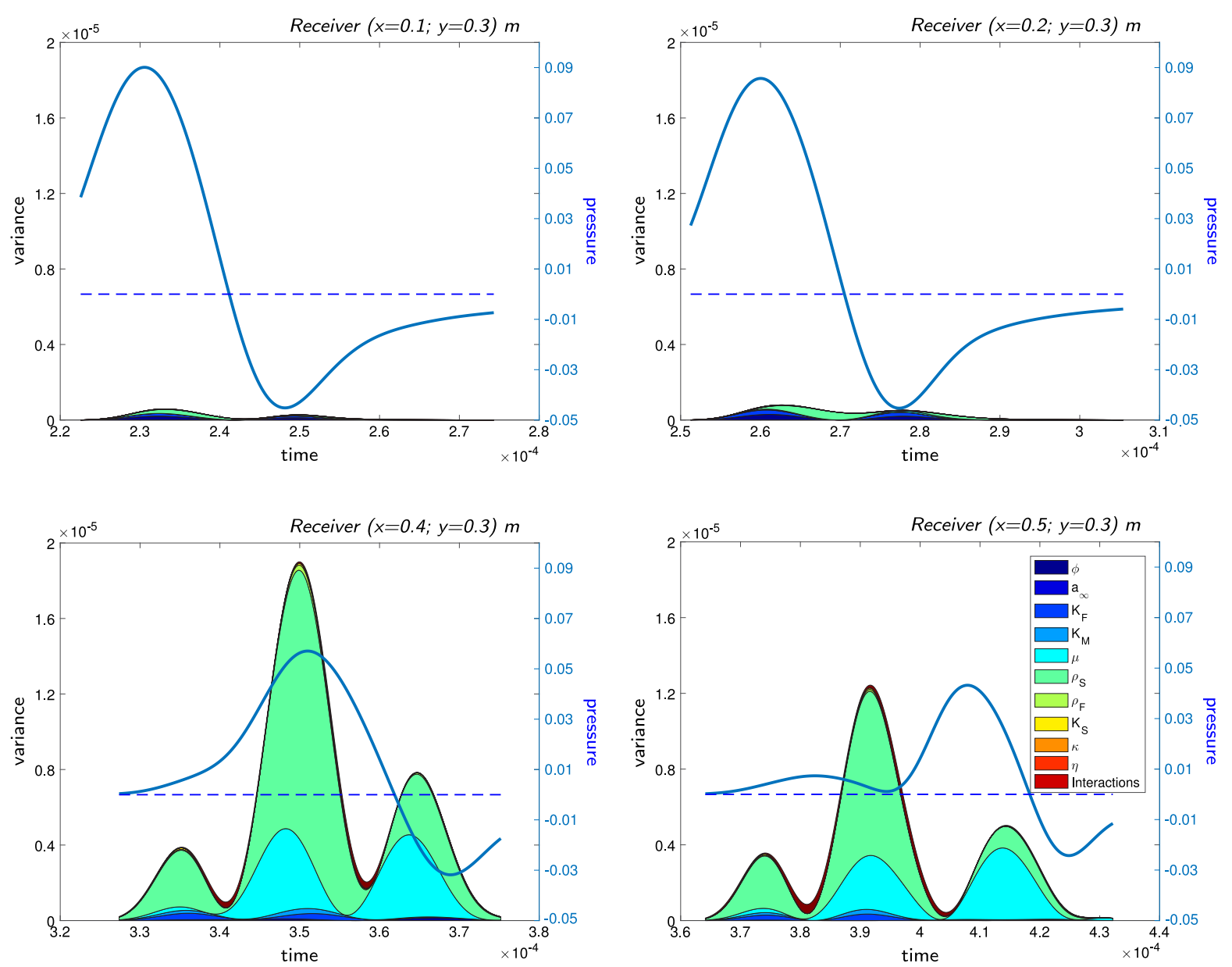

Fig. 5. Full time $(\mathrm{s})$ evolution of the partial variances for fluid pressure $(\mathrm{Pa})$ at: $(x=0.1 ; y=0.3) \mathrm{m}$ (top left); $(x=0.2 ; y=0.3) \mathrm{m}($ top right); $(x=0.4 ; y=0.3) \mathrm{m}$ (bottom left); $(x=0.5 ; y=0.3) \mathrm{m}$ (bottom right). 

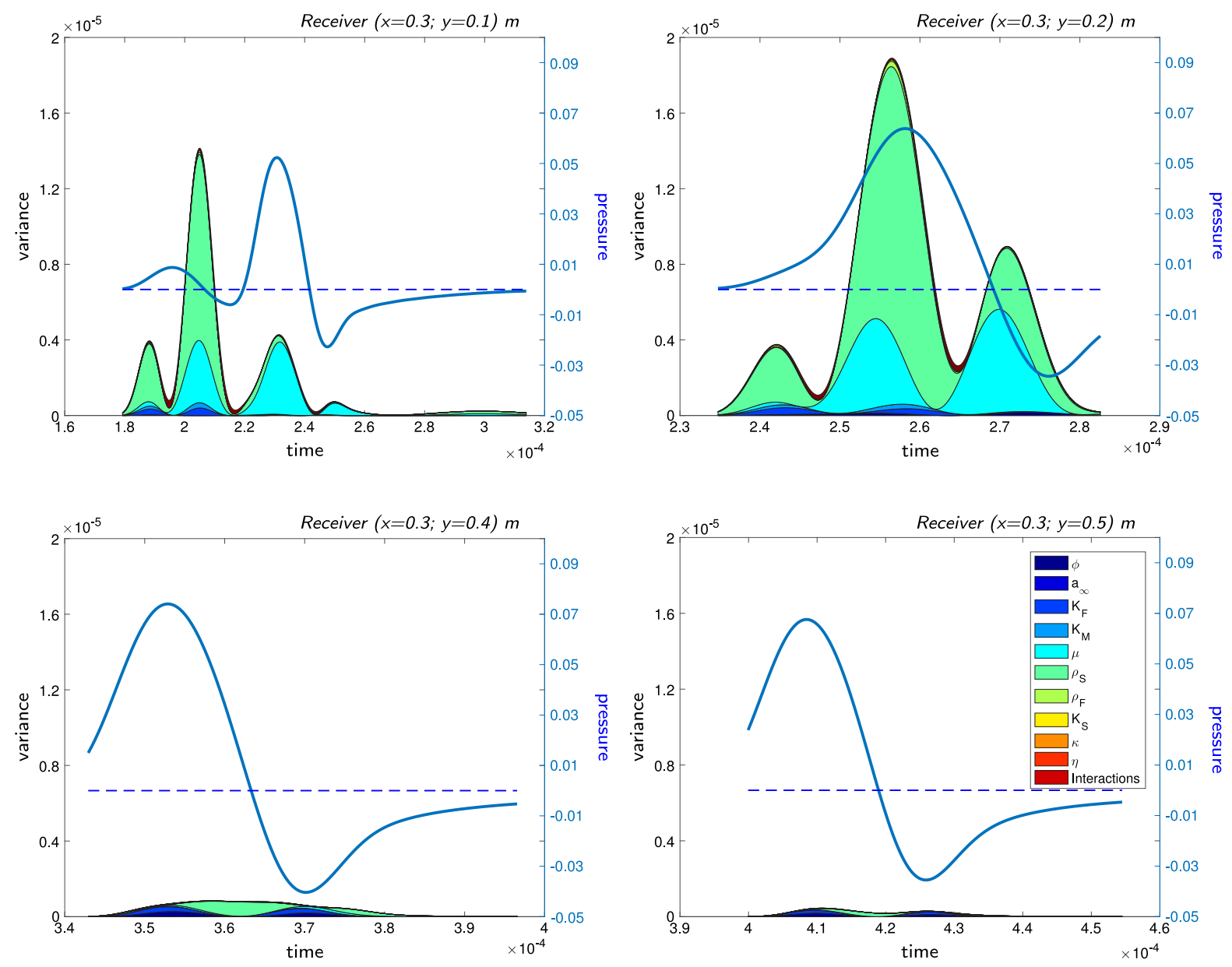

Fig. 6. Full time (s) evolution of the partial variances for fluid pressure (Pa) at: $(x=0.3 ; y=0.1) \mathrm{m}$ (top left); $(x=0.3 ; y=0.2) \mathrm{m}($ top right); $(x=0.3 ; y=0.4) \mathrm{m}$ (bottom left); $(x=0.3 ; y=0.5) \mathrm{m}$ (bottom right).

The total variance varies considerably as a function of both time and space. These fluctuations of the total variance are not linked with those of the pressure mean. For example, in Fig. 5 (bottom left), the maximum values of variance and pressure mean occur at the same time, leading to a standard deviation of $8 \%$ at this time step. In Fig. 5 (bottom right), it is no longer the case and the maximum value of variance appears for a low level of the pressure mean. Consequently, the maximum level of information that can be extracted from observation of fluid pressure on the studied parameters does not always coincide with the maximum of the fluid pressure, as could have been expected. A full waveform inspection of the variance and mean of the signal clearly provides more information than the study at specific given times chosen a priori.

When $x$ increases, the cumulated variance increases and the parts due to parameters $\rho_{S}$ and $\mu$ become preponderant, see Fig. 5. When $x$ decreases, the influence of $\mu$ and $\rho_{S}$ is reduced and fluid parameters $\phi$ and $K_{F}$ remain at the same level, since the influence of the pressure wave comes either directly from the excitation source or after being converted into a surface wave on the surface between the fluid and the poroelastic half spaces. The other parameters are irrelevant. Interaction between the parameters is negligible, except in a narrow zone near the interface.

Fig. 6 confirms that the influence of $\mu$ and $\rho_{S}$ is lower when moving away from the poroelastic surface, due to a strong decreasing impact of the surface wave, and that the influence of both $\phi$ and $K_{F}$ remains stable.

\subsection{Fluid/porous medium interface I}

Fig. 7 (left) presents the time evolution of the partial variances of the pore pressure at the interface between the upper fluid and the porous medium at $(x=0,3 ; y=-0.01) \mathrm{m}$.

The global level of variance explained at the interface by the variation of the selected parameters is more than ten times higher than the maximal one observed in the fluid part. Moreover, the pressure mean value is much lower. The variance reaches a value of $3.10^{-4} \mathrm{~Pa}$, which gives a standard deviation of $1.710^{-2} \mathrm{~Pa}$ compared to the maximum value of the fluid pressure mean of $2.510^{-2} \mathrm{~Pa}$. This corresponds to a $70 \%$ fluctuation for this simulated measure. The signal is divided into 

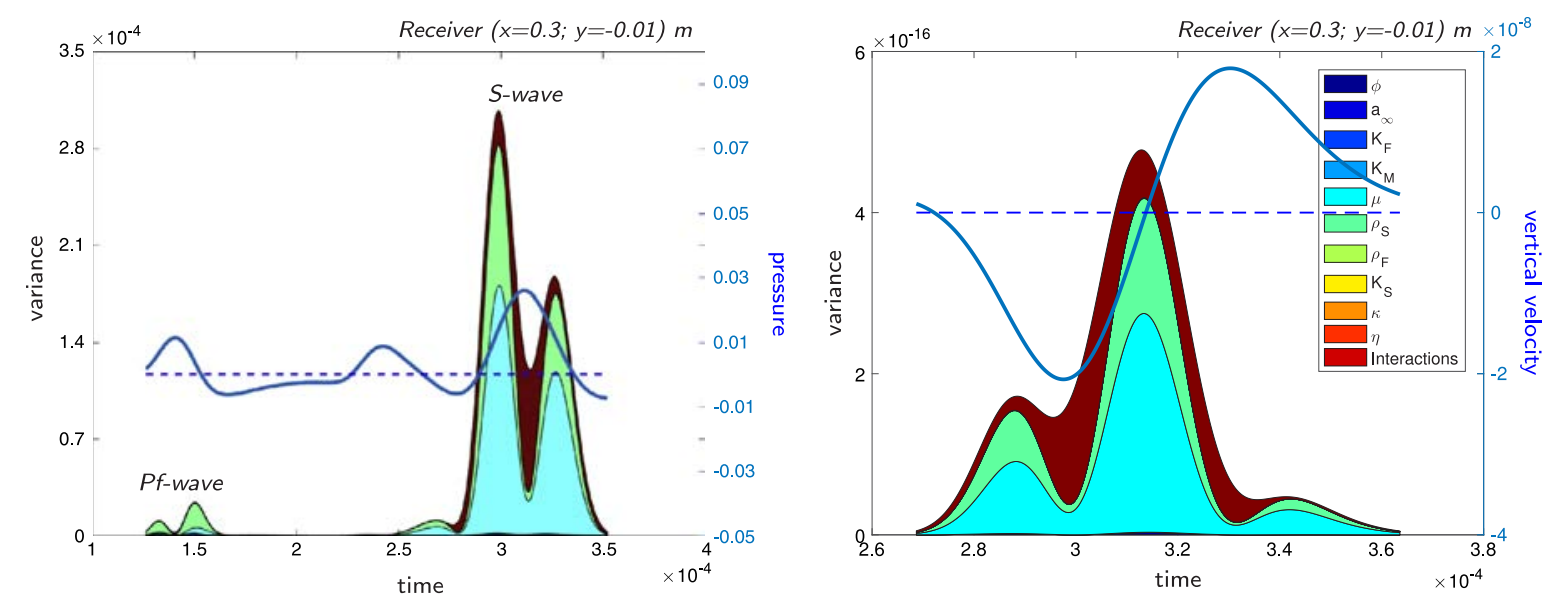

Fig. 7. Full time (s) evolution of the partial variances for: the pore pressure $p(\mathrm{~Pa})(\mathrm{left})$ and the vertical velocity $\dot{u}_{y}(\mathrm{~m} / \mathrm{s})$ (right) at a selected location $(x=0.3, y=-0.01) \mathrm{m}$, near the fluid-porous medium interface $I$.

two parts: the first one corresponds to the fast compressional wave (arrival time centered around $0.14 \mathrm{~ms}$ ) and the second one is due to the shear and surface (of Pseudo-Stoneley type) waves (arrival time estimated after the arrival time of shear wave $S$ at $0.28 \mathrm{~ms}$ ). A factor 2 between the compressional and shear/surface waves is noticed on Fig. 7 (left) whereas the variance is much higher for the surface contribution.

For the time period corresponding to fast compressional wave Pf wave influence, parameters $\rho_{S}$ and $\mu$ are the most relevant ones, and in a fewer level, other parameters $\phi, K_{F}$, and $K_{M}$ also show a small influence. For the time period corresponding to $S$ and surface waves influence, the pressure response is governed by parameters $\rho_{S}$ and $\mu$. The pressure, that does not intrinsically depend on the shear effect, is under its influence owing to the strong mechanical couplings of the Biot theory. As the fluid/porous interface has been considered as an "open-pore" interface, the same sensitivity is observed for a point situated inside the fluid part $\Omega_{F}$ near the interface. Moreover, an interaction (indicated in brown) between the parameters clearly appears. Note that as the influence of $\rho_{S}$ and $\mu$ decreases, the interactions increases. Fig. 8 (top) shows that the interaction only occurs between parameters $\rho_{s}$ and $\mu$.

Fig. 7 (right) presents the time evolution of the partial variances for the same receiver but for the vertical solid velocity. Only the shear and surface wave contribution is seen. Even if the intrinsic value of the variance is low, it yields a standard deviation of similar order of magnitude than this of the pressure, relative to the velocity amplitude. As for the pressure, the variance is entirely linked to variations of parameters $\rho_{S}$ and $\mu$, intrinsically and by their mutual interaction. Indeed, the considerable level of interaction observable on Fig. 7 (right) only implies these two parameters, as confirmed on Fig. 8 (bottom). Other parameters absolutely do not influence the response.

The same trend is observed along the fluid/porous medium interface no matter $x$ (results not shown), except for sensors located near the excitation source where all the body and surface waves are still not decoupled.

\subsection{Porous medium $\Omega_{P}$}

Fig. 9 (left) presents the temporal evolution of the pore pressure partial variances at $(x=0 ; y=-0.3) \mathrm{m}$. We can observe similar levels of pressure variance inside the porous medium. Most relevant parameters are in a hierarchic ranking : (i) $\rho_{S}$, (ii) $K_{F}, K_{M}$ and $\mu$, (iii) $\phi$ and $K_{S}$ in a minor way. Other input parameter effects $\left(a_{\infty}, \kappa, v\right.$ and $\left.\rho_{F}\right)$ are negligible. Unlike in the fluid half-space, interaction occurs for all the influential parameters, see Fig. 10. The analysis of the partial variances of the vertical solid velocity does not give supplementary information (not shown here).

The representation of the pore pressure partial variances directly below the solicitation at $(x=0 ; y=-0.01) \mathrm{m}$ shows different results, Fig. 9 (right). Near the surface and below the solicitation, the most relevant parameters are porosity $\phi$ and fluid bulk modulus $K_{F}$. Fluid-type parameters become preponderant. The influence of permeability is also seen: it appears then that permeability $\kappa$ becomes influential when the sensor is located near the surface and is entirely quasi submitted to a compressional effect. There is no interaction between the parameters.

\subsection{Assessment}

Fig. 11 presents the spatial distribution of the $\log _{10}$ partial variances for the fluid pressure at $T=0.19 \mathrm{~ms}$. It is a complementary way to analyze the results, and it confirms the main results presented in the previous subsections. They are summarized in Table 3. 

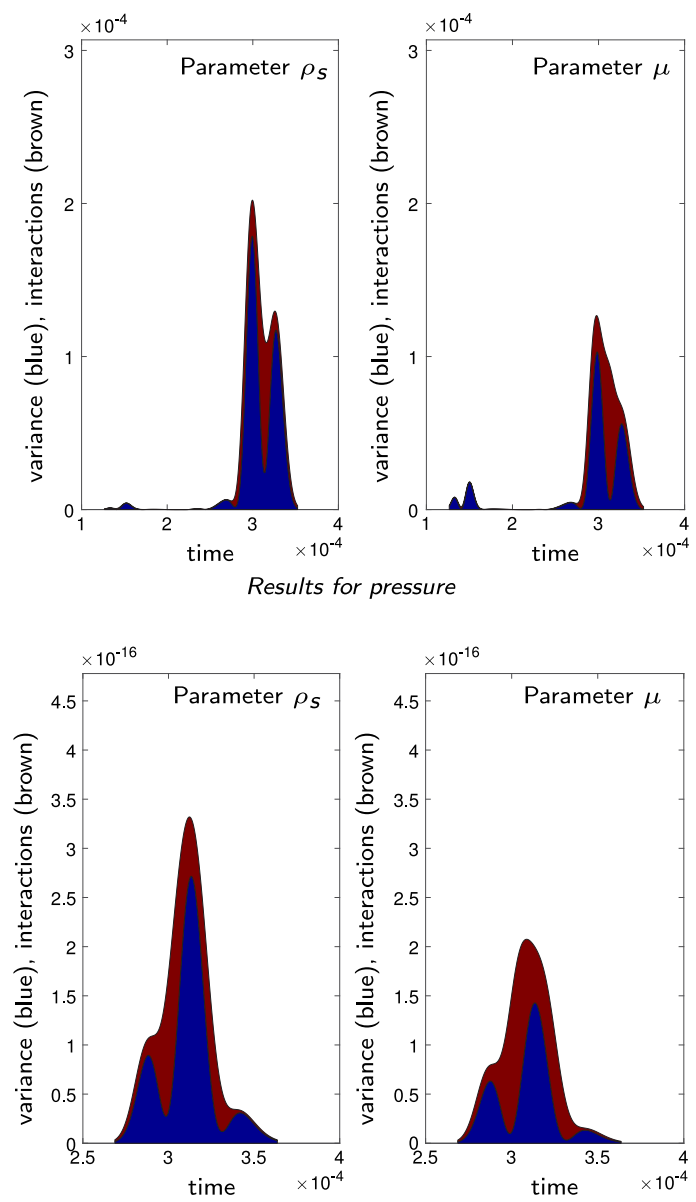

Results for vertical velocity

Fig. 8. Full time (s) evolution of the first order partial variances (in blue) and interaction terms (in brown) for $\rho_{S}$ (left) and $\mu$ (right) for: the pore pressure $p$ (Pa) (top) and the vertical velocity $\dot{u}_{y}(\mathrm{~m} / \mathrm{s})$ (bottom) at a selected location $(x=0.3, y=-0.01) \mathrm{m}$, near the fluid-porous medium interface $I$.
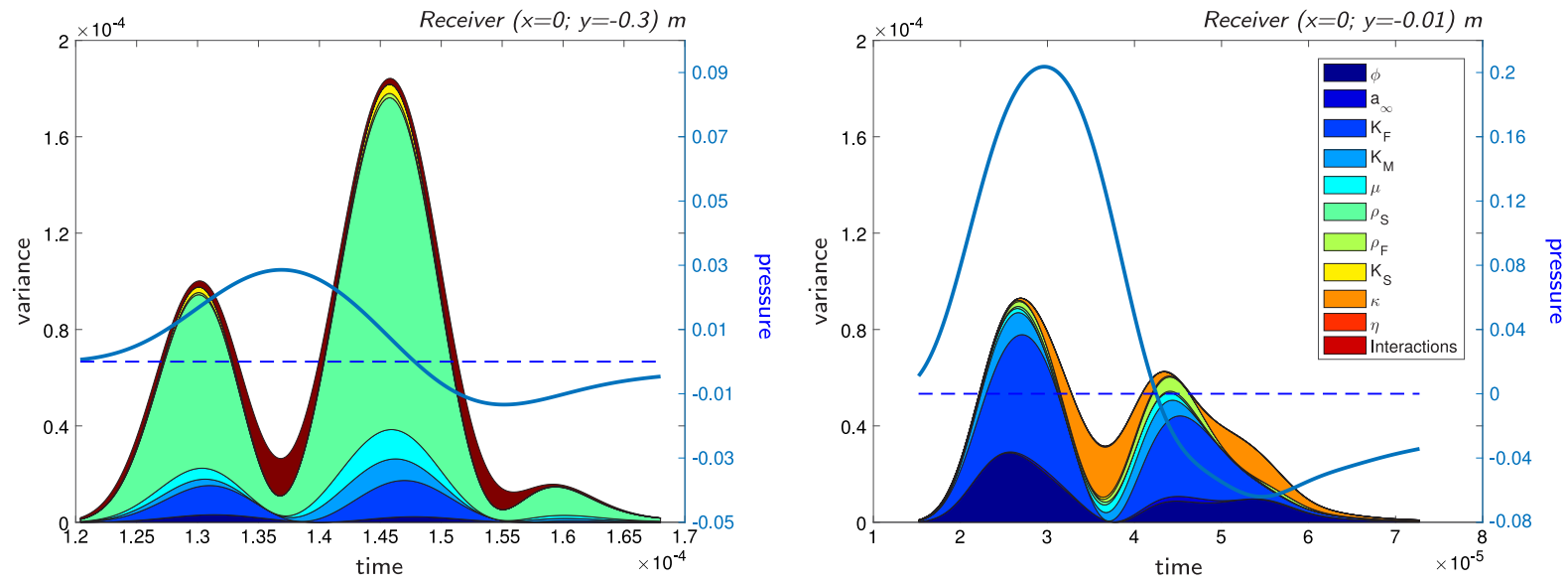

Fig. 9. Full time (s) evolution of the partial variances for the pore pressure $p(\mathrm{~Pa})$ at selected locations: $(x=0, y=-0.3) \mathrm{m}(\mathrm{left})$ and $(x=0, y=-0.01)$ $\mathrm{m}$ (right) belonging to the porous medium $\Omega_{P}$ directly under the solicitation.

For the configuration under study, the main conclusions are:

- a $10 \%$ uniform fluctuation of tortuosity $a$, dynamic viscosity $\eta$, fluid density $\rho_{F}$, and bulk modulus of the skeleton $K_{S}$ has a very small impact on the pressure and vertical velocity responses. Consequently, these parameters will be 

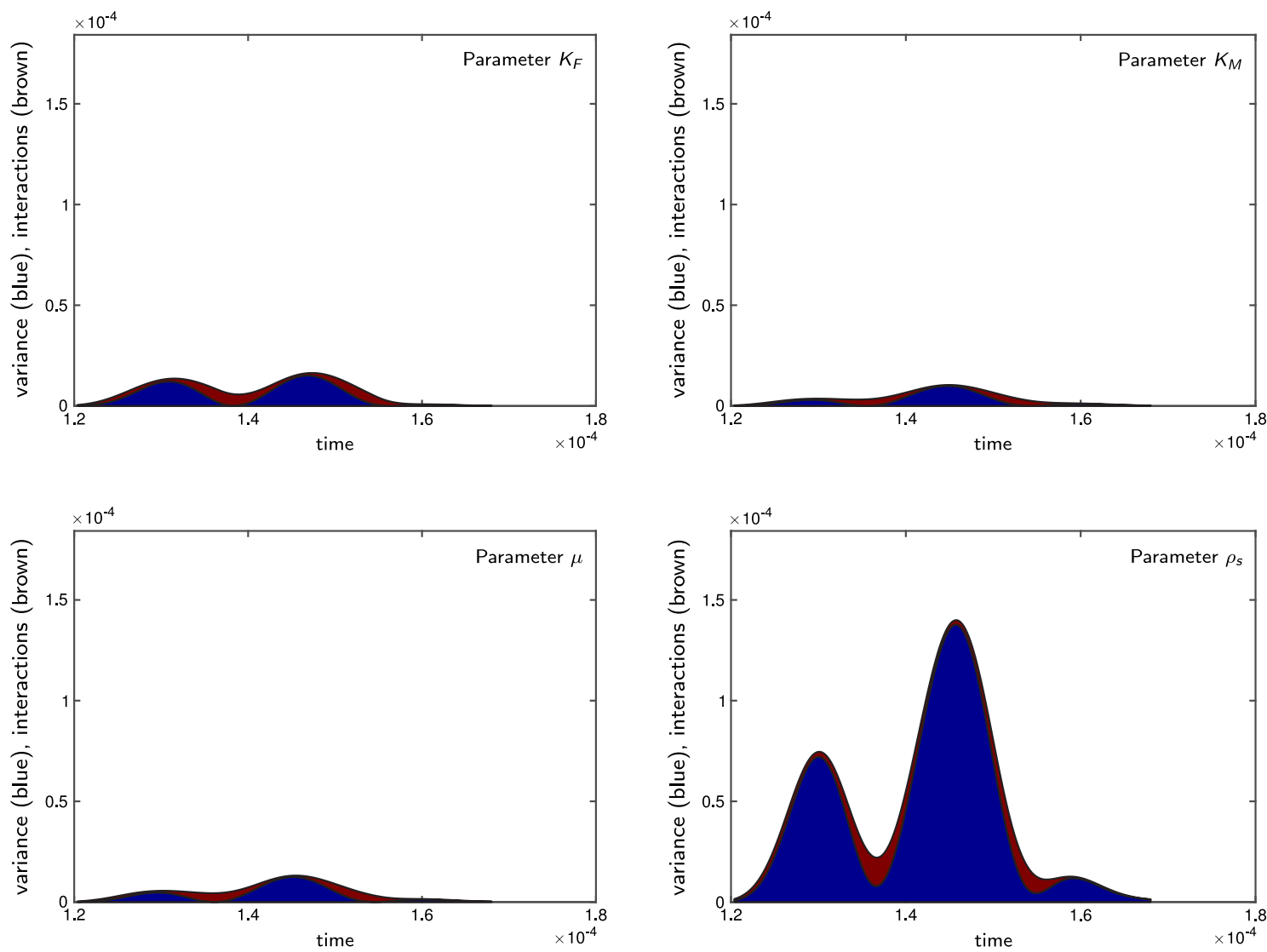

Fig. 10. Full time (s) evolution of the first order partial variances (in blue) and interaction terms (in brown) for $K_{F}$ (top left), $K_{M}$ (top right), $\mu$ (bottom left) and $\rho_{S}$ (bottom right) for the pore pressure $p(\mathrm{~Pa})$ at $(x=0, y=-0.3) \mathrm{m}$.

Table 3

Synthesis of the major parameters of influence.

\begin{tabular}{|c|c|c|c|}
\hline Different parts & Influential parameters & Interaction & Relative Figures \\
\hline$\Omega_{F}$ (pressure) & $\begin{array}{l}\rho_{S}, \phi, K_{F}, \mu \\
\mu \text { influence decreasing for points closer to the source }\end{array}$ & minor/no & $4,5,6$ \\
\hline$I$ (pressure) & $\rho_{S}, \mu$ and $\phi, K_{F}, K_{M}$ & $\begin{array}{l}\text { minor for } P \text { wave, } \\
\text { yes for } S \text { wave }\end{array}$ & 7 (left), 8 (top) \\
\hline$I$ (velocity) & $\rho_{S}, \mu$ & yes & 7 (right), 8 (bottom) \\
\hline \multirow[t]{2}{*}{$\Omega_{P}$ (pressure) } & $\begin{array}{l}\text { (i) mainly } \rho_{S} \\
\text { (ii) then } K_{F}, K_{M}, \mu \\
\text { (iii) and } \phi, K_{S}\end{array}$ & yes & 9 (left), 10 \\
\hline & near pressure field: $\phi, K_{F}, \kappa, \rho_{F}$ & no & 9 (right) \\
\hline
\end{tabular}

difficult to estimate in an inversion process but at the same time their values do not need to be precisely known to obtain reliable results from the model,

- the sensitivity analysis shows that complementary information is contained in the signals function of both the time and the receiver location, because of the different waves traveling:

- the partial variance fluctuation of a mechanical quantity (pressure, velocity) relative to the shear wave passage is greatly linked to the grain parameters of the porous medium $\mu$ and $\rho_{S}$,

- the partial variance fluctuation of a mechanical quantity relative to the fast compressional wave passage can be due to the fluid parameters of the porous medium, such as porosity $\phi$, fluid bulk modulus $K_{F}$ and intrinsic permeability $\kappa$,

- analysis of partial variances shows interaction between parameters, specifically during the shear wave passage and not during the compressional wave passage, 

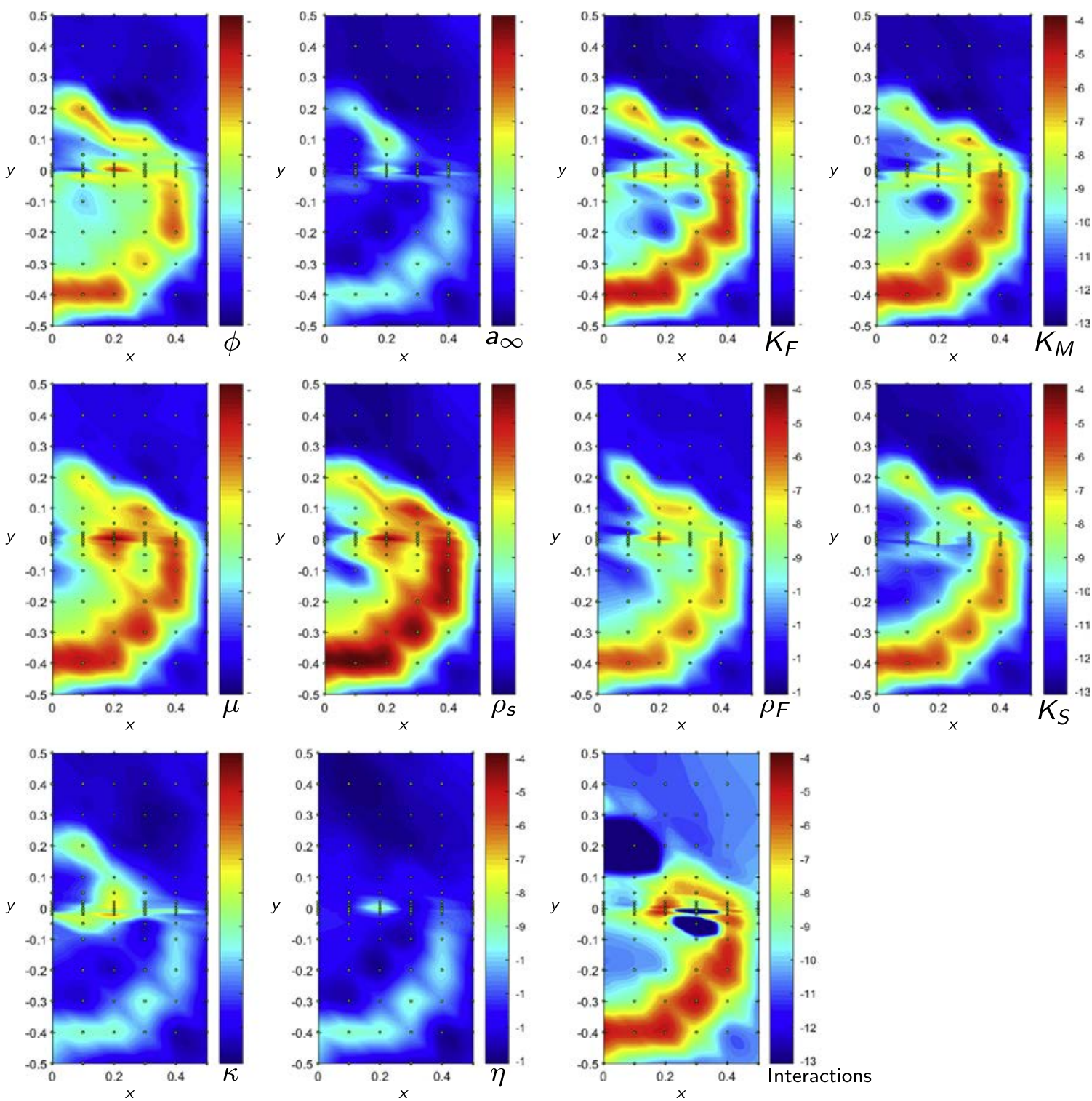

Fig. 11. Spatial distribution ( $\mathrm{m}$ ) of the $\log _{10}$ partial variances for the fluid pressure (Pa) due to uncertainties in $\phi, a_{\infty}, K_{F}, K_{M}, \mu, \rho_{S}, \rho_{F}, K_{S}, \kappa$ and $\eta$ at $T=0.19 \mathrm{~ms} . T=0.19 \mathrm{~ms}$ has been chosen to emphasize the influence of each wave, i.e., to separate clearly the various waves in the media and to make visible non-zero simulated signal in the whole space under consideration This figure has been plotted with the Matlab grid data function from a 156 receiver grid.

- studying the pressure response inside the porous medium or in the fluid part isolates the influence of the compressional waves whereas studying the vertical velocity includes the influence of the shear waves,

- due to surface waves of Pseudo-Stoneley type, the model provides a more complex response at the interface: vertical velocity is governed by parameters $\mu$ and $\rho_{S}$ whereas pressure is governed either by fluid parameters (weak direct shear solicitation), or by solid parameters (strong direct shear solicitation).

It must be emphasized that results obtained with variance-based methods are rarely generic. Paleari and Confalonieri [53] have recently shown the strong influence of factor distributions on the results of a sensitivity analysis conducted with the Sobol method. The conclusions given here are thus likely to be strongly linked to the problem under study: geometry, frequencies involved, kind of material, and choice of uncertainty range. We have chosen to illustrate the potentiality of the EFAST method on an academic case but it is important to understand that a SA has to be led for each particular configuration. 


\subsection{Comments on the application of the SA methodology}

If, as explained above, the results of the application of variance-based methods can hardly be regarded as generic, these methods are themselves clearly generic. They can be applied regardless of:

- the type of model studied; these methods are called model-free, i.e. they do not require any assumption about the shape of the model,

- the uncertainty distribution of the factors studied, provided such distributions are independent and the number of studied factors is not too important.

It however happens in practice that uncertainties about unknown factors depend on each other. It may be the case for example when these uncertainties result from experimental data [54] or when they are constrained by some engineering processes [55]. In this case, new terms appear in the variance decomposition formulation, Eq. (3): the partial variance owing to a given factor may be influenced by its dependence on other inputs making the results of the classical approaches difficult to interpret. Several alternatives have been proposed in the recent years to address this problem, see [56] for a short review.

Estimating partial variances using variance-based methods is computational expensive and thus not appropriate for very high-dimensional problems [14]. In practice, it is advised to apply these methods to a number of factors inferior to about twenty [57]. Although it is not the case in this study, the number of uncertain input factors of complex models usually exceeds this limit. In this case, screening methods are usually applied in a first step to identify the set of important factors on which variance-based method can be eventually applied [58].

\section{Conclusion and further work}

Taking into account, explicitly, the fluid part of a porous material is a laudable goal and has been done in this manuscript by a complete poroelastic approach. However, at the same time, the use of an elaborate model increases the number of parameters, which leads to a difficulty in handling them. Consequently, it is of interest to both use an elaborated model and try to restrict it only to the influential parameters in correlation with the configuration under study. Sensitivity analysis methods have proven in many domains and applications to be efficient tools for exploring model behavior under input parameter uncertainties and complex relationships between model inputs and outputs. However, this kind of analysis is still rarely applied to wave propagation in poroelastic environments.

In this study, we have used a global sensitivity analysis method for studying the simulation of fluid pressure and solid velocity full waveform responses in a configuration representing a fluid overlying a poroelastic medium, subjected to a transient excitation. This method has allowed us to track the most influential parameters. It has been shown to be powerful in highlighting the complementary information contained in the signal function of both the time and the receiver location.

If the methodology proposed here is generic, the results obtained are strongly linked to the characteristics of the problem under study: soil nature and geometry, frequencies under study, and parameter uncertainty distributions. This pleads for a systematic application of such kinds of methods when new configurations are studied. If the EFAST method is relatively costly in terms of number of model simulations required, more computationally efficient methods such as RBD-FAST or metamodelling strategies can be applied for more computationally demanding models.

When considering an inversion process, a preliminary sensitivity analysis can be of great help in the construction of the inversion strategy. For instance, in the example studied, since the interface points are particularly sensitive to the shear Lamé constant and solid bulk density, the inversion can ensure that measurements at the interface have information on these parameters. On the contrary, since near the source, the porosity and fluid bulk modulus become more influential, the inversion process should consider near field response. This will be the next step of our work.

\section{Acknowledgments}

The authors wish to thank the Editor-in-Chief and the two anonymous Reviewers for their constructive comments which helped us to substantially improve the manuscript.

\section{Appendix A. EFAST algorithm}

Saltelli et al. [31] proposed an algorithm to perform EFAST that can be summarized as follows:

(1) Choose an interference factor $M$ and sampling size $N$ so that $N-1$ is a multiple of $4 . M^{2}$

(2) Set $i=1$

(3) Generate $\omega_{i}$ the frequency of factor $X_{i}$ :

$\omega_{i}=\frac{N-1}{2 M}$

(4) Generate $k$ low frequencies a follows:

$\boldsymbol{\omega}_{\sim i}=(1, \Delta+1,2 \Delta+1, \ldots,(k-1) \Delta+1)^{T}$, with $\Delta=$ floor $\left(\frac{N-1}{4 k M^{2}}\right)$

If $\Delta=0, \omega_{\sim i}$ may contain different values but with repetitions (see [31])

(5) Generate randomly $k$ phase-shifts $\left(\varphi_{1}, \ldots, \varphi_{k}\right) \in[0,2 \pi]^{k}$ 
(6) Generate the EFAST sample :

$s_{q}=-\pi+\frac{(2 q-1) \pi}{N}, q=1, \ldots, N$

$x_{q j}=F_{j}^{-1}\left(\frac{1}{\pi} \arcsin \left(\sin \left(\omega_{j} s_{q}+\varphi_{j}\right)\right)+\frac{1}{2}\right), j=1, \ldots, k$ and $q=1, \ldots, N$

(7) For each draw $\mathbf{x}_{q}=\left(x_{q 1}, \ldots, x_{q k}\right)$ with $q=1, \ldots, N$ :

run the model and save the model response of interest $f\left(\mathbf{x}_{q}\right)$

(8) Compute the Fourier coefficients as follows:

$\hat{A}_{l}=\frac{1}{N} \sum_{q=1}^{N} \cos \left(l s_{q}\right) \times f\left(\mathbf{x}_{q}\right)$

$\hat{B}_{l}=\frac{1}{N} \sum_{q=1}^{N} \sin \left(l s_{q}\right) \times f\left(\mathbf{x}_{q}\right)$

$\forall l \in\left\{-\frac{N-1}{2}, \ldots,-1,0,1, \ldots, \frac{N-1}{2}\right\}$

(9) Evaluate the total variance $\hat{V}$ and partial variances $\left(\hat{V}_{i}, \hat{V T}_{i}\right)$

(10) Set $i=i+1$,

(11) If $i>k$ stop, otherwise resume from (3).

As suggested by an anonymous reviewer, this algorithm could be written in an alternative way by initially setting $\Delta=M$ and deducing from its value the list of frequencies, $\boldsymbol{\omega}_{\sim i}$ and $\omega_{i}$, and the sample size $N$. In case the computational cost is not affordable, the value of $\Delta$ could eventually be decreased.

\section{Appendix B. Theoretical equations}

\section{B.1. Source expression}

The source term is expressed as:

$$
S(t)=\left\{\begin{array}{l}
\sum_{m=1}^{4} a_{m} \sin \left(\beta_{m} \omega_{C} t\right) \quad \text { if } 0<t<\frac{1}{f_{C}} \\
0 \text { otherwise }
\end{array}\right.
$$

where $\beta_{m}=2^{m-1}$ and $\omega_{C}=2 \pi f_{C}$; the central frequency $f_{C}=20 \mathrm{kHz}$. The coefficients $a_{m}$ are: $a_{1}=1, a_{2}=-21 / 32$, $a_{3}=63 / 768, a_{4}=-1 / 512$ [17]. The Fourier transform of $S(t)$ is explicitly given by:

$$
S^{*}(\omega)=\sum_{m=1}^{4} a_{m} \frac{\beta_{m} \omega_{C}}{2 \pi} \frac{\exp \left(i 2 \pi \omega / \omega_{C}\right)-1}{\omega^{2}-\beta_{m}^{2} \omega_{C}^{2}}
$$

\section{B.2. Acoustic equations for the fluid medium}

The acoustic equations are written as follows:

$$
\left\{\begin{array}{l}
p=-K_{F} \nabla \cdot \mathbf{U} \\
\Delta p-\frac{\rho_{F}}{K_{F}} \ddot{p}=-S(t) \delta(x) \delta\left(y-y_{s}\right)
\end{array}\right.
$$

with $\delta$ the Dirac distribution and $\ddot{p}=\partial^{2} p / \partial t^{2}$. The parameters and variables are defined in the main text, see Section 4.2.

\section{B.3. Biot model for the poroelastic medium}

The low-frequency Biot model is valid at frequencies below the critical value defined as:

$$
f_{c}=\frac{\eta \phi}{2 \pi a_{\infty} \kappa \rho_{F}}
$$

Based on the constitutive equations and the conservation of momentum in porous media, one obtains [47-49]:

$$
\left\{\begin{array}{l}
\boldsymbol{\Sigma}=\left(\left(K_{M}-\frac{2}{3} \mu\right) \nabla \cdot \mathbf{U}-\left(1-\frac{K_{M}}{K_{S}}\right) p\right) \mathbf{I}+2 \mu \boldsymbol{\Xi} \\
p=-\left(\frac{1-\phi-\frac{K_{M}}{K_{S}}}{K_{S}}+\frac{\phi}{K_{F}}\right)^{-1}\left(\left(1-\frac{K_{M}}{K_{S}}\right) \nabla \cdot \mathbf{u}+\nabla \cdot \mathbf{w}\right) \\
\nabla \boldsymbol{\Sigma}=\left(\phi \rho_{F}+(1-\phi) \rho_{S}\right) \ddot{\mathbf{u}}+\rho_{F} \ddot{\mathbf{w}} \\
-\nabla p=\rho_{F} \ddot{\mathbf{u}}+\frac{a_{\infty} \rho_{F}}{\phi} \ddot{\mathbf{w}}+\frac{\eta}{\kappa} \dot{\mathbf{w}}
\end{array}\right.
$$

where $\mathbf{u}$ is the solid displacement, $\mathbf{U}=\left(U_{x}, U_{y}\right)^{T}$ is the fluid displacement, $\mathbf{w}=\phi(\mathbf{U}-\mathbf{u})=\left(w_{x}, w_{y}\right)^{T}$ is the relative displacement, $\mathbf{I}$ is the identity tensor, $\boldsymbol{\Sigma}$ is the stress tensor, $\boldsymbol{\Xi}=\frac{1}{2}\left(\boldsymbol{\nabla} \mathbf{u}+\nabla^{T} \mathbf{u}\right)$ is the strain tensor, and $p$ is the pore pressure. The mechanical parameters are defined in the main text, see Section 4.2. 


\section{B.4. Interface conditions}

The interface condition between the fluid half-space and the porous medium can be described using the following conditions:

$$
[p]_{0}=0 ;\left(\sigma_{y y}\right)_{0}^{-}=-(p)_{0}^{+} ;\left(\sigma_{x y}\right)_{0}^{-}=0 ;\left(u_{y}\right)_{0}^{-}+\left(w_{y}\right)_{0}^{-}=\left(U_{y}\right)_{0}^{+},
$$

where $[p]_{0}=(p)_{0}^{+}-(p)_{0}^{-}$denotes the jump in the fluid pressure from $\Omega_{F}$ to $\Omega_{P}$ across $y=0$, and $(p)_{0}^{+}=\lim _{\varepsilon \rightarrow 0^{+}} p(x, \varepsilon, t)$.

\section{Appendix C. Main characteristics of the semi-analytical approach}

To solve the partial differential systems issued from the fluid upper part and from the porous medium, we use a semianalytical method, detailed in [18], and whose main steps, in the present configuration, are summarized as follows:

- in the porous medium, Helmholtz decompositions provides a $(\mathbf{u}, \mathbf{w})$ second-order wave formulation,

- applying $x$ and $t$ Fourier transforms yields decoupled ordinary differential systems in the frequency-wavenumber domain, associated with fast and slow compressional waves $P_{f}$ and $P_{s}$, and with shear wave $S$,

- the pressure field in the fluid upper part is developed analytically in the frequency-wavenumber domain,

- the interface conditions between the fluid and porous medium are taken into account to couple the two parts,

- the global system provides the solutions in terms of displacements or stresses/pressure in the frequency-wavenumber domain,

- to finish, inverse transforms over the horizontal wavenumber and the frequency are performed numerically to obtain displacement, stress, and pressure fields in the time and space domain.

The integrand over the horizontal wavenumber shows fast oscillatory behavior and the envelope of the maximum amplitudes shows sharp peaks. Consequently, an alternative technique to the usual Fast Fourier Transform for evaluating the integral properly has been chosen, based on the Filon quadrature, which is particularly accurate and suitable for dealing with these integrals. The Filon quadrature has been specifically adapted to carefully treat the sharp changes in the integrand occurring around the wavenumbers of the propagating waves : the wavenumbers are calculated and sorted out to divide the entire integration interval into several parts. The quadrature is then performed by discretizing finely in the neighborhood of these wavenumbers and more coarsely farther away. The integral is truncated depending on the highest wavenumber and adapted to each frequency.

Numerical integration over the frequency variable is done using a Simpson quadrature with a regular grid.

\section{References}

[1] L. De Barros, M. Dietrich, B. Valette, Full waveform inversion of seismic waves reflected in a stratified porous medium, Geophys. J. Int. 182 (3) (2010) $1543-1556$

[2] C. Morency, Y. Luo, J. Tromp, Acoustic, elastic and poroelastic simulations of CO2 sequestration crosswell monitoring based on spectral-element and adjoint methods, Geophys, J. Int. 185 (2) (2011) 955-966.

[3] N.P. Chotiros, An inversion for Biot parameters in water-saturated sand, (Ph.D. thesis), J. Acoust. Soc. Am. 112 (2002) $1853-1868$.

[4] R. Sidler, Reflection of Seismic Waves from Attenuating and Anisotropic Ocean Bottom Sediments, ETH Zurich, 2008.

[5] P.M. Gauzellino, J.M. Carcione, J.E. Santos, S. Picotti, A rheological equation for anisotropic - anelastic media and simulation of field seismograms, Wave Motion 51 (5) (2014) 743-757.

[6] Y. Bouzidi, D.R. Schmitt, Incidence-angle-dependent acoustic reflections from liquid-saturated porous solids, Geophys. J. Int. 191 (2012) $1427-1440$.

[7] J.L. Buchanan, R.P. Gilbert, M.J. Yo, Recovery of the parameters of cancellous bone by inversion of effective velocities, and transmission and reflection coefficients, Inverse Problems 27 (12) (2011) 125006.

[8] N. Sebaa, Z.E. Fellah, M. Fellah, E. Ogam, A. Wirgin, F.G. Mitri, C. Depollier, W. Lauriks, Ultrasonic characterization of human cancellous bone using the Biot theory:inverse problem, J. Acoust. Soc. Am. 120 (4) (2006) 1816-1824.

[9] A. Curtis, A. Lomax, Prior information, sampling distributions and the curse of dimensionality, Geophysics 66 (2001) 372-378.

[10] S. Aoi, T. Iwata, K. Irikura, F.J. Sanchez-Sesma, Waveform inversion for determining the boundary shape of a basin structure, B. Seismol. Soc. Am. 85 (5) (1995) $1445-1455$.

[11] T. Scotti, A. Wirgin, Reconstruction of the three mechanical material constants of a lossy fluid-like cylinder from the low-frequency scattered acoustic fields, C. R. Mécanique 332 (2004) 717-724.

[12] B. Dupuy, Propagation des Ondes Sismiques dans les Milieux Multiphasiques Hétérogènes : Modélisation Numérique, Sensibilité et Inversion des Paramètres Poroélastiques, (Ph.D. thesis), University Grenoble, 2011.

[13] G. Lefeuve-Mesgouez, A. Mesgouez, E. Ogam, T. Scotti, A. Wirgin, Retrieval of the physical properties of an anelastic solid half space from seismic data, J. Appl. Geophys. 88 (2013) 70-82.

[14] P. Wei, Z. Lu, J. Song, Variable importance analysis: A comprehensive review, Reliab. Eng. Syst. Saf. 142 (2015) 399-432.

[15] S. Tarantola, N. Saint-Geours, Editorial, Reliab. Eng. Syst. Saf. 134 (2015) 239-240.

[16] A. Saltelli, M. Ratto, T. Andres, F. Campolongo, J. Cariboni, D. Gatelli, M. Saisana, S. Tarantola, Global Sensitivity Analysis. the Primer, Wiley, 2008.

[17] G. Lefeuve-Mesgouez, A. Mesgouez, G. Chiavassa, B. Lombard, Semi-analytical and numerical methods for computing transient waves in 2D acoustic/poroelastic stratified media, Wave Motion 49 (7) (2012) 667-680.

[18] A. Mesgouez, G. Lefeuve-Mesgouez, Transient solution for multilayered poroviscoelastic media obtained by an exact stiffness matrix formulation, Int. J. Numer. Anal. Methods Geomech. 33 (2009) 1911-1931.

[19] L. De Barros, M. Dietrich, Perturbations of the seismic reflectivity of a fluid-saturated depth-dependent poro-elastic medium, J. Acoust. Soc. Am. 123 (2008) 1409-1420.

[20] I.M. Sobol, Sensitivity estimates for nonlinear mathematical models, Math. Model. Comput. Exper. 1 (1993) 07-414. 
[21] H. Rabitz, O.F. Alis, General foundations of high-dimensional model representations, J. Math. Chem. 25 (2-3) (1999) $197-233$.

[22] T. Homma, A. Saltelli, Importance measures in global sensitivity analysis of nonlinear models, Reliab. Eng. Syst. Saf. 52 (1) (1996) 1-17.

[23] A. Saltelli, S. Tarantola, On the relative importance of input factors in mathematical models: safety assessment for nuclear waste disposal, J. Amer. Statist. Assoc. 97 (2002) 702-709.

[24] L. Li, Z. Lu, D. Wu, A new kind of sensitivity index for multivariate output, Reliab. Eng. Syst. Saf. 147 (2016) $123-131$.

[25] R.I. Cukier, C.M. Fortuin, K.E. Shuler, A.G. Petschek, J.H. Schaibly, Study of the sensitivity of coupled reaction systems to uncertainties in rate coefficients, I. Theory, J. Chem. Phys. 59 (1973) 3873-3877.

[26] R.I. Cukier, J.H. Schaibly, K.E. Shuler, Study of the sensitivity of coupled reaction systems to uncertainties in rate coefficients. III Analysis of the Approximations, J. Chem. Phys. 63 (1975) 1140-1149.

[27] J.H. Schaibly, K.E. Shuler, Study of the sensitivity of coupled reaction systems to uncertainties in rate coefficients. II Applications, J. Chem. Phys. 59 (1973) 3879-3888.

[28] R.I. Cukier, H.B. Levine, K.E. Shuler, Nonlinear Sensitivity Analysis of multiparameter model systems, J. Comput. Phys. 26 (1978) 1-42.

[29] M. Koda, G.J. McRae, J.H. Seinfeld, Automatic sensitivity analysis of kinetic mechanisms, Int. J. Chem. Kinet. 11 (1979) $427-444$.

[30] G.J. McRae, J.W. Tilden, J.H. Seinfeld, Global sensitivity analysis - a computational implementation of the Fourier Amplitude Sensitivity Test (FAST), Comput. Chem. Eng. 6 (1982) 15-25.

[31] A. Saltelli, S. Tarantola, K.P.S. Chan, Quantitative model-independent method for global sensitivity analysis of model output, Technometrics 41 (1999) 39-56.

[32] Y. Lu, S. Mohanty, Sensitivity analysis of a complex, proposed geologic waste disposal system using the Fourier Amplitude Sensitivity Test method, Reliab. Eng. Syst. Saf. 72 (2001) 275-291.

[33] S. Fang, G.Z. Gertner, S. Shinkareva, G. Wang, A. Anderson, Improved generalized Fourier amplitude sensitivity test (FAST) for model assessment, Statist. Comput. 13 (2003) 221-226.

[34] C. Xu, G.Z. Gertner, Extending a global sensitivity analysis technique to models with correlated parameters, Comput. Statist. Data Anal. 51 (12) (2007) 5579-5590.

[35] C. Xu, G.Z. Gertner, A general first-order global sensitivity analysis method, Reliab. Eng. Syst. Saf. 93 (7) (2008) $1060-1071$.

[36] S. Tarantola, D. Gatelli, T.A. Mara, Random balance designs for the estimation of first order global sensitivity indices, Reliab. Eng. Syst. Saf. 91 (2006) 717-727.

[37] S. Tarantola, M. Koda, Improving random balance designs for the estimation of first order sensitivity indices, Procedia Soc. Behav. Sc. 2 (6) (2010) $7753-7754$.

[38] T.A. Mara, Extension of the RBD-FAST method to the computation of global sensitivity indices, Reliab. Eng. Syst. Saf. 94 (8) (2009) $1274-1281$.

[39] J.Y. Tissot, C. Prieur, Bias correction for the estimation of sensitivity indices based on random balance designs, Reliab. Eng. Syst. Saf. 107 (2012) 205-213.

[40] A. Saltelli, J.H. Anderes, T. Homma, Sensitivity analysis of model output - an investigation of new techniques, Comput. Statist. Data Anal. 15 (1993) 211-238.

[41] A. Saltelli, R. Bolado, An alternative way to compute Fourier amplitude sensitivity test (FAST), Comput. Statist. Data Anal. 26 (1998) 445-460.

[42] D. Makowski, C. Naud, M.H. Jeuffroy, A. Barbottin, H. Monod, Global sensitivity analysis for calculating the contribution of genetic parameters to the variance of crop model prediction, Reliab. Eng. Syst. Saf. 91 (2006) 1142-1147.

[43] C. Xu, G.Z. Gertner, Understanding and comparisons of different sampling approaches for the Fourier Amplitudes Sensitivity Test (FAST), Comput. Statist. Data Anal. 55 (1) (2011) 184-198.

[44] S. Buis, D. Wallach, S. Guillaume, H. Varella, P. Lecharpentier, M. Launay, M. Guerif, J.E. Bergez, E. Justes, The STICS crop model and associated software for analysis, parameterization and evaluation, in: Laj R. Ahuja, Liwang Ma (Eds.), in: Methods of Introducing System Models into Agricultural Research. Advances in Agricultural Systems Modeling, vol. 2, Am. Soc. Agron., Crop Sc. Soc. Am., Soil Sc. Soc. Am., Madison, 2011 , pp. $395-426$.

[45] G. Pujol, B. Iooss, A. Janon with contributions from K. Boumhaout, S. Da Veiga, J. Fruth, L. Gilquin, J. Guillaume, L. Le Gratiet, P. Lemaître, B. Ramos, T. Touati, F. Weber, Sensitivity: Global Sensitivity Analysis of Model Outputs. R package version 1.12.2, 2016. https://CRAN.R-project.org/package= sensitivity.

[46] S. Marino, I.B. Hogue, C.J. Ray, D.E. Kirschner, A methodology for performing global uncertainty and sensitivity analysis in systems biology, J. Theoret. Biol. 254 (1) (2008) 178-196.

[47] M.A. Biot, Theory of propagation of elastic waves in a fluid-saturated porous solid. I: Low-Frequency Range, J. Acoust. Soc. Am. 28 (2) (1956) 168-178.

[48] T. Bourbié, O. Coussy, B. Zinszner, Acoustics of Porous Media, Gulf Publishing Company, 1987.

[49] J.M. Carcione, Wave Fields in Real Media: Wave Propagation in Anisotropic, Anelastic, Porous and Electromagnetic Media, Elsevier, 2007.

[50] J.H. Rosenbaum, Synthetic microseismograms: logging in porous formation, Geophysics 39 (1) (1974) 14-32.

[51] S. Feng, D.L. Johnson, High-frequency acoustic properties of a fluid/porous solid interface, I. New Surface Mode. J. Acoust. Soc. Am. 74 (3) (1983) 906-914.

[52] A.I.M. Denneman, G.G. Drijkoningen, D.M.J. Smeulders, K. Wapenaar, Reflection and transmission of waves at a fluid/porous medium, Geophysics 28 (2002) 2-291.

[53] L. Paleari, R. Confalonieri, Sensitivity analysis of a sensitivity analysis: we are likely overlooking the impact of distributional assumptions, Ecol. Model. 340 (2016) 57-63.

[54] E. Borgonovo, S. Tarantola, Moment independent and variance-based sensitivity analysis with correlations: An application to the stability of a chemical reactor, Int. J. Chem. Kinet. 40 (2008) 687-698.

[55] W. Hao, Z. Lu, P. Wei, Uncertainty importance measure for models with correlated normal variables, Reliab. Eng. Syst. Saf. 112 (2013) 48-58.

[56] G. Chastaing, F. Gamboa, C. Prieur, Generalized hoeffding-sobol decomposition for dependent variables-application to sensitivity analysis, Electron. J. Stat. 6 (2012) 2420-2448.

[57] B. Iooss, P. Lemaître, A review on global sensitivity analysis methods, in: Uncertainty Management in Simulation-Optimization of Complex Systems, Springer, 2015, pp. 101-122.

[58] R. Confalonieri, Monte Carlo based sensitivity analysis of two crop simulators and considerations on model balance, Eur. J. Agro. 33 (2) (2010) 89-93. 